\title{
The Tsunami of Cardiometabolic Diseases: An Overview
}

\section{Gundu HR Rao}

Laboratory Medicine and Pathology; Director, Thrombosis Research, Lillehei Heart Institute, University of Minnesota

\author{
"Correspondence author \\ Gundu HR Rao \\ Director \\ Laboratory Medicine and Pathology \\ Thrombosis Research \\ Lillehei Heart Institute \\ University of Minnesota \\ 12500 Park Potomac Ave \\ Unit 306N \\ Maryland, 20854. USA \\ E-mail : gundurao9@gamil.com
}

Submitted : 26 Nov 2019 ; Published : 4 Jan 2020

\begin{abstract}
Cardiometabolic diseases, including obesity and Type-2 diabetes, are a growing concern and have become an epidemic worldwide. A World Health Organization (WHO) report, - 'Global Prevalence of Type-2 diabetes' estimates incidence of this disease, to be $9 \%$ among adults. An estimated 1.5 million death worldwide, were directly caused by diabetes. More than $80 \%$ of the Type-2 diabetes-related deaths occur in low-and middle-income countries. Worldwide in 2013, it was estimated that almost 400 million people suffer from diabetes, and this is supposed to reach a high of 600 million by 2035. According to the World Health Organization estimates, the impact of Type-2 diabetes-related health care expenditure, to prevent and manage diabetes and its clinical complications in the USA alone, was USD 360 billion in 2010 and will exceed 360 billion by 2030. WHO predicts losses in national income from Diabetes and Cardiovascular Disease (CVD) to be in equivalents of 557 billion in International Dollars (ID) in China, 303 billion in Russia, and 236 billion in India. Currently, there are 75 million diabetics in India and an equal number of pre-diabetics. The incidence of Type-2 diabetes in China also has reached epidemic proportions. Gulf Countries also have reported a high incidence of diabetes. One of the Global targets for diabetes management was to halt by 2025, the rise in the age-standardized adult prevalence of this disease, at its 2010 levels. However, according a recent publication in the Lancet (April 2016) by the NCD Risk Factor Collaboration, if the post-2000 trends continue in the increased incidence of type-2 diabetes, the probability of meeting the global target of halting the rise in the prevalence of diabetes by 2025 at the 2020 level worldwide is lower than $1 \%$ for men and women. Because of these observations, the best choice we have is, to go by the World Diabetes Federation recommendations, and manage the hyperglycemia efficiently. Besides, develop programs for early detection of altered glucose metabolism, and implement robust strategies for normalization of this altered state. In conclusion, through robust prevention programs, reduce the disease incidence, through better diagnostic tests, detect early risks that initiate or promote clinical complications, and by effective management of the risks, reduce or prevent acute events related to the end-organ failure.
\end{abstract}

Keywords: Cardiometabolic diseases, obesity, type-2 diabetes, activity trackers, wearables, thermal imaging

\section{Introduction}

Metabolic diseases such as hypertension, excess weight, obesity, type -2 diabetes, and vascular diseases have reached epidemic proportions worldwide [1-20]. November 14th is the 'World Diabetes Day' according to the International Federation of Diabetes. The theme for 2018 and 2019 is 'The Family and Diabetes'. Creating global awareness is one of the primary goals. Emphasizing the need for a healthy lifestyle also is an important goal. November 17th is celebrated as World
Prematurity Day. It is important to understand the relations between the prematurity, low birth weight, intrauterine growth retardation, and the increase in the incidence and prevalence of metabolic diseases. Both the World events. - diabetes and prematurity day are celebrated in November, we can consider this month a global awareness month for early detection and prevention of cardiometabolic diseases. 
The rapid increase in the prevalence of cardiometabolic diseases is described as "Tsunami's" in the literature. These natural catastrophic events are unpredictable. Contrary to this situation, the epidemic of cardiometabolic diseases is predictable, and from time to time professional public health organizations, have done population-level surveys and published their assessments and predictions [2-6, 21]. Despite these reports, call for action, white papers, and prevention guidelines, metabolic diseases are on the rise worldwide. Twin epidemics of diabetes and obesity, contribute significantly to the morbidity and mortality of cardiovascular and cerebrovascular diseases. Diabetes has increased two-fold and obesity by four-fold in the last three decades, worldwide. A few years ago, India was considered the diabetes capital and now China has beaten India with this dubious distinction. When we consider the number of prediabetics, China, India and the USA has a greater number of prediabetics than diabetics. This is the target population, who are 'at risk' for developing full-blown diabetes in a decade or much earlier. American Heart Association held its first Cardiometabolic Health and Diabetes Summit on December 5th, 2017 in Dallas, Texas. The primary objective was to identify and prioritize opportunities, to improve cardiometabolic health and diabetes care in the United States [22].

International Diabetes Federation says, "Over fifty percent of type-2 diabetes is preventable." World Diabetes Day serves as the world's largest awareness campaign, reaching over 1billion people in more than 160 countries. Because of the fact, that World Prematurity Day also is celebrated this month, we would like to discuss some aspects of prematurity and its role in the development of metabolic diseases, later in life. Holdsworth Memorial Mission Hospital, Mysore, India, has kept birth records of all the children born in that hospital since 1934. Indian Council of Medical Research (ICMR), established a "birth cohort study" in 1969, at five regional centers in India. In 1993, the Medical Research Council of the UK established an Epidemiology Resource Center at the Mission Hospital Mysore, and initiated a program to assess the importance of early life on diseases in this 'Mysore Cohort'. These studies as well as other studies conducted in the UK, demonstrated the relationship of low birth weight (LBW) with elevated blood pressure, insulin resistance, obesity, diabetes, coronary artery disease and stroke [22-36]. Based on his observations and collaborative research, David Barker a British epidemiologist, proposed that adverse nutrition in early life, including prenatally as measured by birth weight, increased susceptibility to the metabolic syndrome, which includes obesity, diabetes, insulin sensitivity, hypertension, hyperlipidemia and complications that include coronary artery disease [37]. More than $30 \%$ of the children born in India are of low birth weight. Therefore, we must prevent maternal malnutrition not only for the mother's and baby's health but also for the baby's future health in adulthood.

A news release from the Children's Hospital, Washington DC, says, "The work that children's National Health System physician-scientist Robert Freishtat and colleagues are doing, could soon be a 'game-changer', -when it comes to early intervention and prevention of obesity-related illness. We already know, that there's a direct relationship between the amount of visceral adipose or belly fat a person has, and the development of some of the most common and life-threatening complications of obesity, including cardiovascular disease, and the insulin resistance that leads to diabetes. What remained unclear, until recently, were the precise mechanism for how the increase in belly fat, triggers the onset of additional disease." These findings prompted us, to contact Dr. Robert Freishtat and explore possibilities for developing a bilateral US-India research project, on the role of maternal exosome (miRNA) in reprogramming the fetal genetic material and gene expression. Since the Diabetes group at the King Edwards Memorial (KEM) Hospital Pune, had established a large bio-bank of fetal and maternal tissues, we negotiated with Professor C. S. Yajnik, a working arrangement for preliminary studies. We were also able to find a third partner, Genotypic Technology at Bengaluru, India, who could perform the needed miRNA assays at a short notice. Encouraging results from these preliminary studies helped the team secure, funding's for further studies, from the prestigious National Institutes of Health (NIH), USA [38].

Excess weight can greatly affect one's health in many ways, with type-2 diabetes (T2D) being one of the most serious. When an individual predisposed to diabetes has excess weight, the cells in the body become less sensitive to the insulin. There is some evidence that fat cells are more resistant to insulin than muscle cells. Prime examples include Barker's observations of fetal origin of adult disease (FOAD), the role of protein kinase $\mathrm{C}$-epsilon in fat cell metabolism, and observations related to adiposity-related disorders [39, 40]. Populationbased data suggest, that pediatric obesity is being followed by an increase in type- 2 diabetes, particularly in adolescents and minority groups. Change in the BMI during and after adolescence is the most important predictive variable of adult obesity. Furthermore, oxidative stress, chronic inflammation, endothelial dysfunction, and subclinical atherosclerosis maybe some of the pathophysiological mechanisms, explaining the increased risk of atherosclerotic CVD and diabetes associated with obesity. Obesity has been spreading rapidly throughout the world. Following in its wake is type-2 diabetes, which will affect half a billion people worldwide by 2030 [41]. Swedish researchers involving over 62,000 Danish individuals have demonstrated that childhood overweight is associated with an increased risk for type-2 diabetes in adulthood $[42,43]$.

A consortium of researchers from the USA, Belgium, and Germany suggest that governments, in concert with the private sector, need to set policies that promote healthy nutritional and agricultural policies, favor modifications in the environment that encourage physical activity, and make prevention affordable for all citizens at high risk [44]. The United Nations (UN) Millennium Declaration, signed in September 2000 by the 189 members of the UN, agreed to try to achieve Millennium Development Goals (MDG) by 2015. One of the MDG's was by 2030 , to reduce by one third, premature mortality from noncommunicable diseases, through prevention and treatment. Despite these suggestions and lofty goals, no country has 
reduced, reversed, or prevented an increase in the incidence and prevalence of obesity and type- 2 diabetes. By and large, diabetes is managed worldwide by interventions that reduce blood sugar levels. International diabetes federation in its 2017 Clinical Practice Recommendation suggests monitoring 2- hour postprandial glucose (PPG) after glucose loading. Recommended PPG is $160 \mathrm{mg} / \mathrm{dl}$. Despite such recommendations in the majority of clinics and doctor's offices just fasting blood glucose or hemoglobin A1c (HBA1c) are routinely monitored for the management of diabetes.

We and others have articulated in our earlier reports, that early diagnosis of the risks and robust management of the risks is the only choice for preventing obesity and diabetes-related clinical complications, as well as premature CVD mortality [45-47]. Without any positive action, 1.9 million people worldwide, will remain at risk from the poor health outcomes associated with overweight, obesity, and diabetes. Syndemic of cardiometabolic diseases is a complex topic, and we will not be able to cover all aspects of these lifestyle disorders. We will briefly discuss some salient findings, discuss the role of integration of emerging technologies in early diagnosis of risks and effective management of the risks of metabolic diseases.

\section{Discussion}

In a recent article on the global agenda for the prevention of type-2 diabetes, William Herman writes, "Lifestyle interventions are safe and effective for preventing diabetes, are associated with improved quality of life, and are costeffective, Metformin is effective and safe and cost-effective. National and international efforts are needed to identify at-risk individuals and to systematically apply these interventions" [48]. Except in advanced countries, in the majority of the countries, people do not participate in primary prevention programs, as such the diabetes is detected more or less when some clinical complications develop. Because of this fact, in countries with large populations screening individuals 'at risk' seems to be an expensive proposition. Except for the unrepeatable success story of dramatic reductions in CVD mortality in North Karelia, Finland, no country has designed are successfully executed the prevention of metabolic diseases at the national level [49]. In the absence of such a strategy, we are left with novel approaches, aimed at improving the lifestyle of the individuals, as suggested by the global agenda. Metabolic diseases are by and large lifestyle diseases, and as such every attempt should be made to provide individuals, with the best choices available to improve their quality of life. Because of this point of view, we will discuss some emerging technologies, that may play a significant role in empowering individuals with healthy living.

Two major contributors to wellness are physical activity and a healthy diet. The World Health Organization (WHO), recommends 150 min of moderate-intensity physical activity each week for adults and $60 \mathrm{~min}$ for children and adolescents. However, $25 \%$ of adults and more than $80 \%$ (Latest issue of Lancet) of adolescents, do not achieve the recommended physical activity targets. Results from the Tromso Study, the longest-running population study in Norway, shows that only
$30.4 \%$ of women and $22.0 \%$ of men reach the recommended target [50-52]. Even in an advanced country like the USA, the statistics are no better. As the market for activity trackers has grown, demand for wearables has become increasingly popular. There are more than 500 unique wearables on the market. The most common sensors used in these devices include PPG, GPS, gyroscope, magnetometer, barometer or altimeter. The top five vendors include Fitbit, Xiaomi, Apple, Garmin, and Samsung. Digital health technologies, like mobile apps and wearable devices, can gather data outside of a hospital or clinic. This data includes information about physical activity, sleep, weight, heart rate, nutrition, and water intake. "Collecting real-world, real-time data through digital technologies, will become a fundamental part of the program," said Eric Dishman, director of the 'All of Us' Research Program. The All of Us Research Program (NIH-funded) has launched the Fitbit Bring-your-own-Device (BYOD) project. All of Us participants with any Fitbit device who wish to share Fitbit data with the program may go on to the All of Us participant portal at https://participant.joinallofus.org. Launched in May 2018, All of Us seeks to enroll one million or more participants, to improve the ability to prevent and treat disease, based on individual differences in lifestyle, environment, and genetics. This program is considered a high technology initiative in Landmark All of Us Research Program [53].

Because of the importance of such a large population-based study envisaged by the prestigious Scripps Research Institute and the NIH to track the nation's health, there is a growing interest in the use of noninvasive diagnostic technologies, to empower the end-users, in improving the quality of life. Since a considerable amount of data generated by such a device is dependent on proprietary software analytics and algorithms, we feel that there is a great need for clinical validation of such devices. India was the first country to approve the FreeStyle Libre Pro Flash professional-use device (ambulatory glucose monitor; AMG or Continuous Glucose Monitor; CGM), which uses a patient-worn sensor to track interstitial glucose levels for up to 14 days. Data from the device can be wirelessly downloaded in about 5 seconds. Initially, the device was validated for use by Type- 1 diabetes patients was validated for use by Type- 1 diabetes patients.

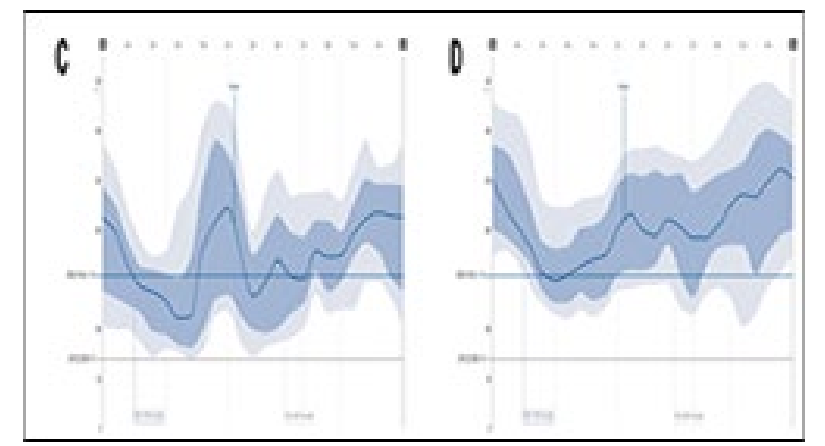

Figure 1: Interstitial Glucose Profiles. (Courtesy: Abbott Diabetes Care, India).

We have validated the device in patients with type- 2 diabetes. These devices record glucose values every 15 minutes, capturing 1340 glucose readings over 14 days. Ambulatory 
glucose graphs are presented in Fig 1. Users can easily understand when sugar levels go high and low, identify postprandial sugar levels. Such real-time information, helps people with diabetes see the impact of food, medication, health, and exercise on their blood sugars. The recent Abbott Sugar Survey conducted in partnership with the Association of Physicians of India showed that $90 \%$ of people surveyed with uncontrolled diabetes (HBA1c higher than 7) in India, continue to perceive that they have control over their glucose levels, despite facts suggesting otherwise. We have described briefly, a minimally invasive technology to continuously monitor glucose levels. Similar ambulatory devices are already in the market for blood pressure monitoring. Despite the availability of such a device, they are not used by the clinicians for monitoring the ambulatory blood pressure of patients. The use of such devices should become a major part of the preventive medicine strategy. In the USA individuals with Medicare insurance can get the CGM sensors for monitoring their glucose profiles. As a part of a primary prevention strategy, devices such as ambulatory blood pressure monitors, ambulatory glucose monitors, and metabolic risk monitors, should be made available for risk assessment and risk management.

Noninvasive diagnostic tools under clinical validation

We have been validating minimally invasive and noninvasive diagnostic tools in India, Sweden, and the USA. We would like to add other noninvasive, simple to use diagnostic devices, to this approach, for monitoring cardiometabolic diseases, for risk stratification, risk management, and prevention strategies (LD-Products, Miami, Florida, USA: www.ldteck.com). We are in the process of screening and validating, a variety of non-invasive diagnostic platforms, for integrating emerging technologies, for the development of affordable healthcare. Figure 2 shows LD- technology products, used for simple tests, that provide data on a variety of biomarkers, which include; height, weight, body mass index, systolic and diastolic pressure, oxygen saturation, body temperature, heart rate, insulin sensitivity, immune response, recovery capacity, fat mass control, mental stress, artery condition, lipid control and an overall wellness score. Since it takes just a few minutes to perform these tests, the device can be used at a variety of settings for general purpose screening. Dr. Albert Maarek and his team at the LD-Technology, Miami, Florida, also have developed more sophisticated non-invasive diagnostic platforms, for the diagnosis and management of cardiometabolic risks.

\section{$\underline{\text { LD Products }}$}

\section{SUDO PATH SYSTEM HARDWARE AND SOFTWARE TO TEST SUDOMOTOR FUNCTION}

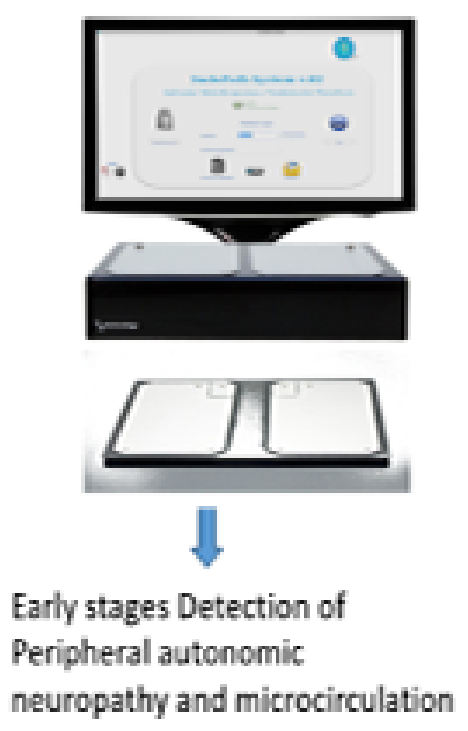

- TM OXI SYSTEM HARDWARE AND SOFTWARE TO TEST AUTONOMIC NERVOUS SYSTEM AND ENDOTHELIAL FUNCTION
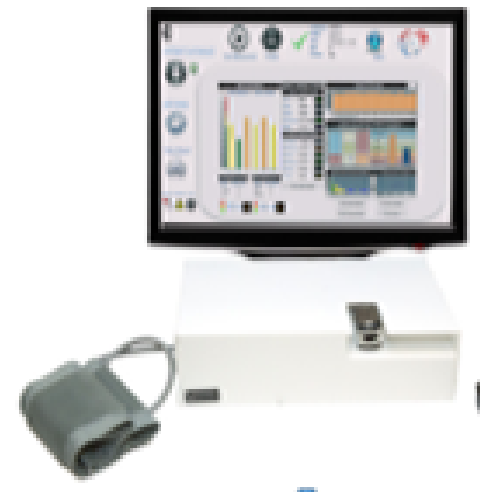

Diabetic or Cardiac Autonomic Neuropathy and endothelial Function evaluation
ES COMPLEX TSS MANAGING SUDOPATH \& TM OXI HARDWARE
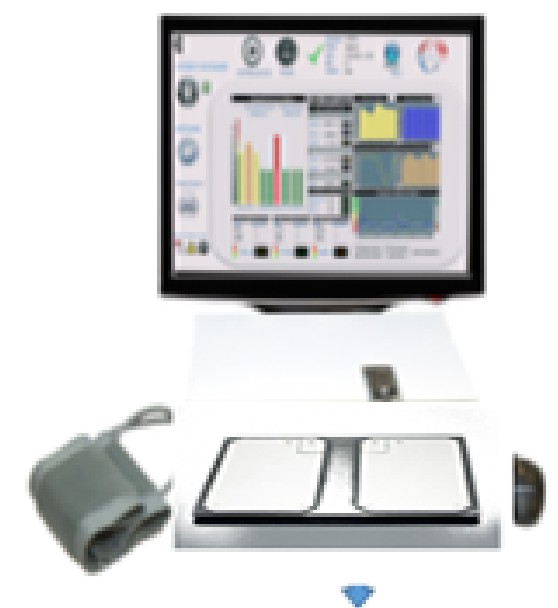

Focus on Diabetes treatment management and early detection of diabetes complications

Figure 2: Noninvasive Diagnosis Tools for Monitoring Cardiometabolic Risks (Courtesy: Dr. Albert Maarek, LD-Technologies, Miami, Florida). 
We have tested the earlier version of their products, SudoPath system, TM Oxi system, and ES Complex system in Mumbai, India, under the leadership of Dr. Pratiksha Gandhi, Chairwoman, IPC Heartcare, Mumbai (Fig 2). Over 1000 patients were studied using this system for early cardiometabolic risk assessment, and therapy management [54-57]. This screening platform, uses oximeter, blood pressure monitor, and a galvanic skin response monitor. Electrical output generated by various tests is computed, integrated, and analyzed by proprietary software and algorithms, to obtain values that represent various clinical markers (risks) for cardiometabolic diseases. Since the tests give assigned values in digital and graphic forms (Fig 3), one can use these tests for early diagnosis of the cardiometabolic risks, as well as for the management of these risks with various interventions, such as lifestyle changes, including diet, and physical activity.

\section{PATIENT PROFILE: RISK FACTORS CHART AND FOLLOW UP}

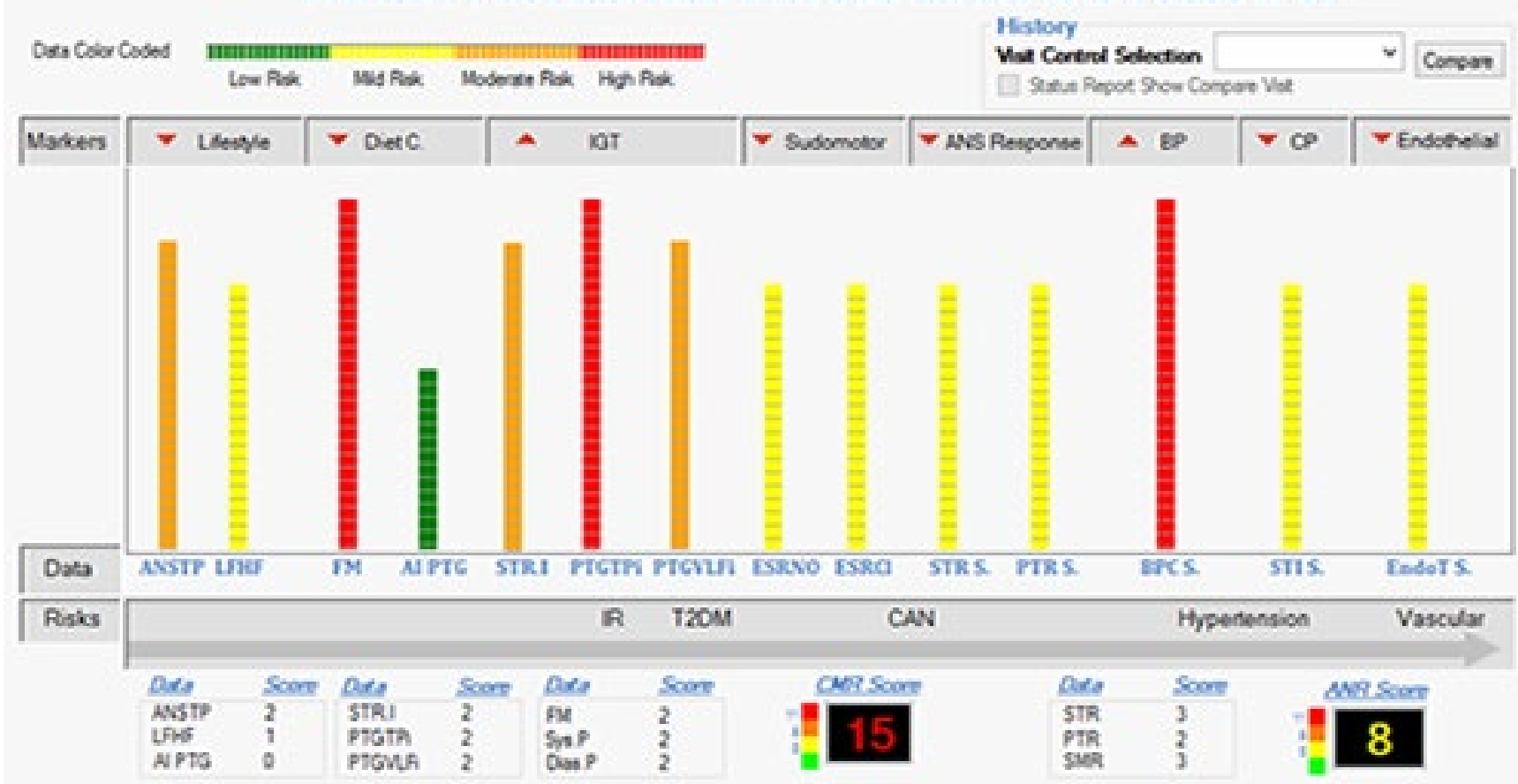

Figure 3: Cardiometabolic Risk Factor Profiling. ( Courtesy: Dr. Albert Maarek, LD-Technology, Miami, Florida)

This system is used in India by Life Span Diabetes Clinics (Lifespan India), under a different name called R.I.S.C Tests. They claim, that these series of tests, provide information on over forty metabolic risks. However, we feel that there is a great need for clinical validation for specificity, accuracy, and efficacy of each of the biomarkers, the system is supposed to detect

LD PRODUCTS 2018 - 2019
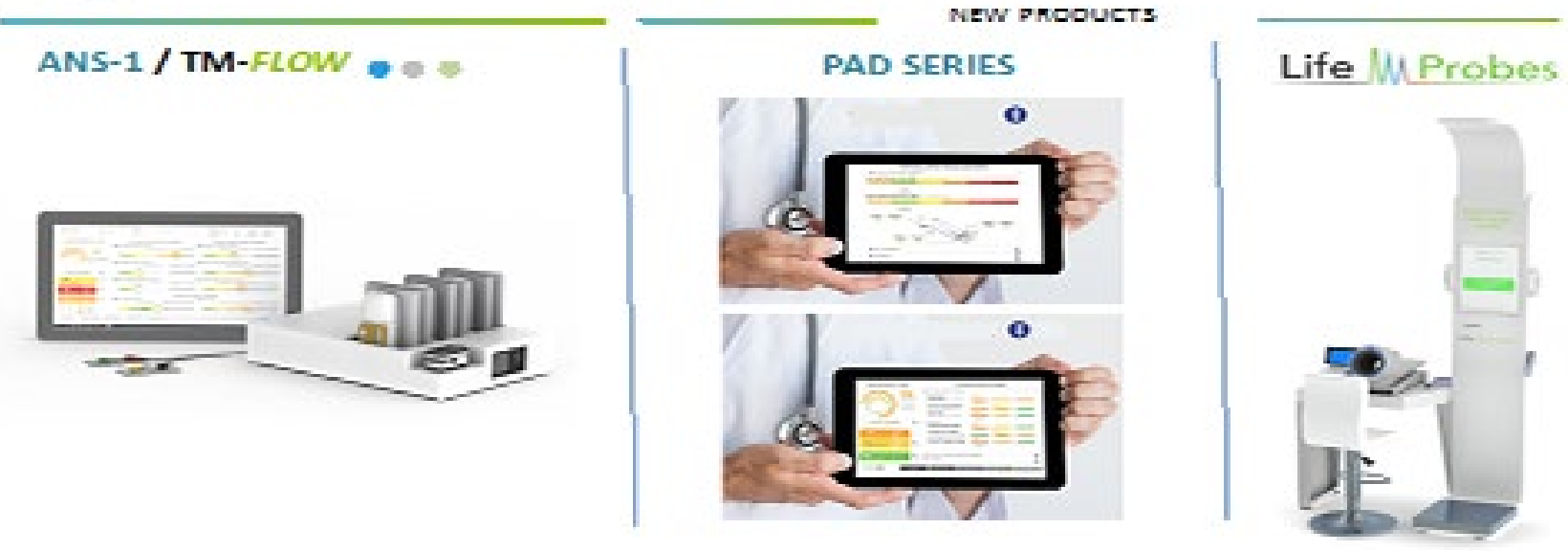

Figure 4: Noninvasive diagnostic Tools. LD-Technology Products ( 2019 Version) . 
The 2019 version of LD products use similar earlier technologies, such as galvanic skin response, for monitoring small artery neuropathy, photoplethysmography for monitoring endothelial dysfunction and cardiac autonomic dysfunction, bio-impedance for assessing body composition, brachial and ankle volumes plethysmography for assessing peripheral artery disease. Using the new version of these devices, we have followed wellness profiles of patients, before and after lifestyle interventions. We are validating this technology in the USA and Sweden for wellness screening.

\section{Emerging Noninvasive Diagnostic Technologies}

Alterations in the vessel wall physiology and compliance of the vessels and the changes if any, in the blood flow velocity, are the earliest stages of vascular dysfunction that could be detected. There are several devices available in the market, that can monitor changes in the flow velocity and provide information on endothelial dysfunction. Some of the devices in use include CV Profiler (Hypertension Diagnostics TM of USA: hypertensiondiagnostics.com), Periscope (Genesis Medical System, Hyderabad, India: genesismedicals.com) and TM-Oxi (LD Technologies, Florida: www.ldteck.com). The majority of the people who suffer heart attacks have no symptoms, making prevention very difficult. However, now with the availability of these devices, we will be able to identify heart disease (vessel wall disease or dysfunction) at its earliest stage and in people with no symptoms. In spite of the advances made in the diagnostic medical device development, we still do not have a simple hand-held pointof-care monitor for diagnosis and management of vascular dysfunction. There is considerable interest in the development of such diagnostic capabilities in the wearables. Apple Watch, for instance, can detect not only heartbeat variability at the wrist, but also perform ECG measurements at a fingertip.

Smartphone apps have been developed to monitor oxygen saturation, heart rate, as well as simple ECG recordings. We have described earlier in this article, the power and novelties of software and algorithm derived measurements of biomarkers (LD Technology Products). Shortly, we will see the development of smartphone-based measurements, for the detection and management of endothelial dysfunction. We have been working on the development of wearables with piezoelectric flexible sensors for monitoring pulse waveforms at various pulse points. We would like to develop simple cost-effective pulse wave monitors, to look at the regional flow of blood and compute the altered flow dynamics with proprietary analytics and algorithms. Yet another possibility to explore will be, to use specially designed ultrasound monitors, to follow blood flow in peripheral arteries and veins, for monitoring flow alterations due to the development of subclinical atherosclerosis or hardening of the arteries.

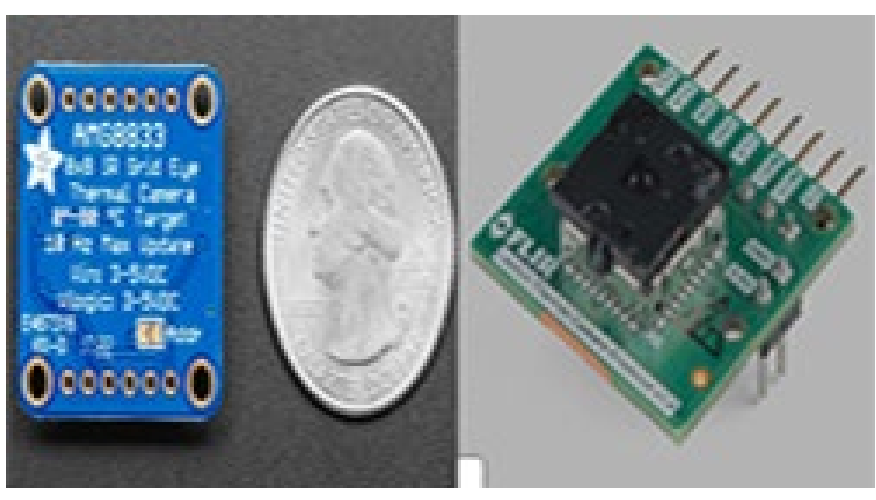

Figure 5: Adrafruit AMG8833IR Sensor

Spark Fun FLIR Sensor for Smart Phone (Source: Internet)

An alternate technology worth exploring seems to be, FLIR based thermal mapping. The acronym FLIR, refers to forward-looking infrared. FLIR Systems is the world's largest commercial company, specializing in the design and production of thermal imaging cameras, components, and imaging sensors. FLIR Systems, sells consumer and commercial smartphone cameras. Thermal imaging is in use by Space, Defense, and security segments. However, newer applications in emerging markets, are exploring the use of this technology in various other diagnostic segments. FLIR One family of cameras, use a pair of sensors and FLIR's MSX technology, to produce thermal images that are easier to decipher. FLIR One Pro has incredible temperature range. The resolution on FLIR's Lepton therm sensor, is $90 \times 60$ pixels on the FLIR One. On the other hand, The FLIR One Pro has 160x120 pixels, which gives a much crisp representation of an object's thermal property.
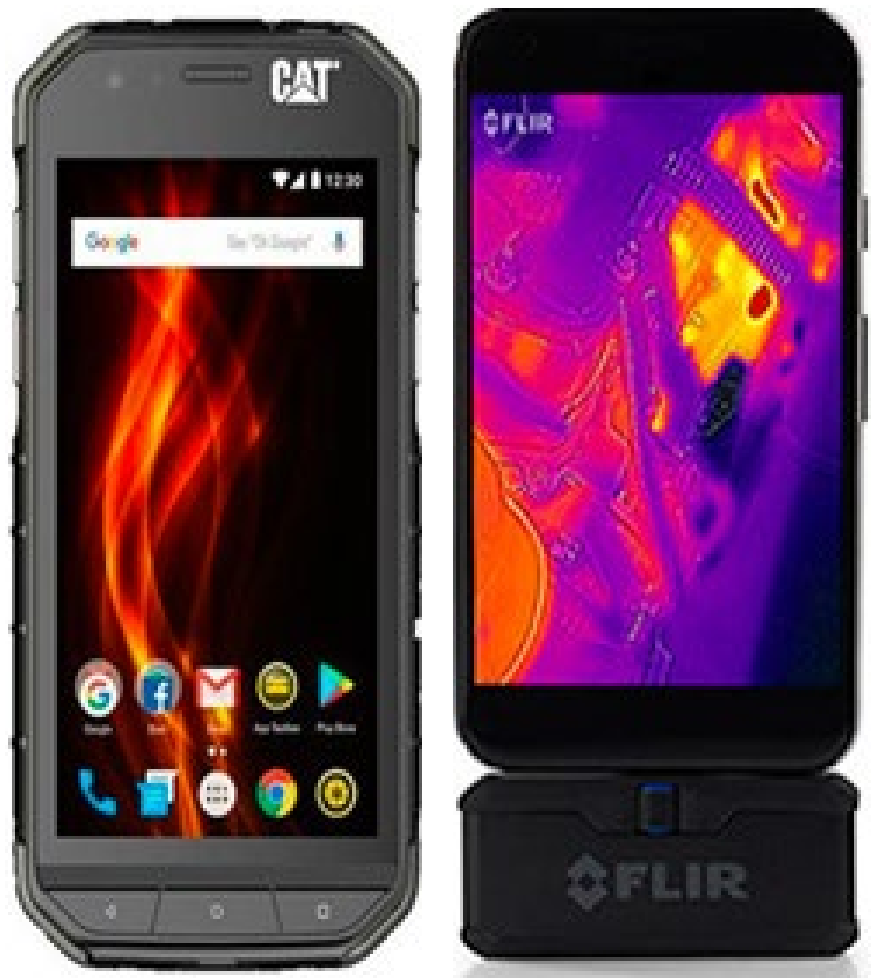

Fig 6. FLIR One Smart Phone Cameras (Source:Internet). 
Fundus autofluorescence imaging has been effectively used as a noninvasive tool for the detection of calcium emboli as well as atherosclerotic plaques in the retinal artery. Near-infrared imaging modality is being widely used for the detection of lipid content in coronary plaques. These two simple noninvasive techniques, fundus autofluorescence, and infrared fundus imaging, have been used for detecting asymptomatic or atypical emboli-even before vascular occlusion occurs [58]. Diabetesrelated clinical complications are by and large mediated by altered flow dynamics and resulting tissue and nerve damage. Peripheral neuropathy is the most common complication of diabetes. In patients with diabetes, feet and legs are the first to get affected, later followed by hands and arms. There seems to be a great opportunity for developing thermography or infrared imaging modalities for the detection of loss of circulation and associated neuropathy, by assessing mean temperatures, temperature differences, and recovery indices after a cold stress test [59]. We have been experimenting with a variety of simple technologies for monitoring blood flow in patients with diabetes. We will report our findings soon after we have standardized these methodologies for clinical applications.

Maternal and neonatal nutrition, intrauterine growth retardation, childhood and adolescent obesity, unhealthy lifestyle contribute significantly to the increased incidence of type-2 diabetes and subsequent development of vascular diseases. Acute events related to vascular diseases are the number one cause of morbidity and mortality worldwide. INTERHEART study done in 52 countries as well as a major Harvard study, have demonstrated the benefits of robust management of modifiable risk factors, in reducing premature mortality due to CVDs $[60,61]$. Diabetes management to a large extent is limited to the management of blood sugar levels. Having said that, it is important to consider the serious clinical implications of diabetes in causing end-organ failure. By careful management, one can prevent the development of diabetes-related complications such as peripheral neuropathy, nephropathy, retinopathy, and various vasculopathies. In this brief overview, we have discussed some emerging technologies available, to empower patients for self-management of the risks associated with this disease. We also have discussed some novel approaches, for the development of cost-effective devices to monitor vascular flow, which plays a very important role in the precipitation of clinical complications, associated with uncontrolled or poorly controlled diabetes.

\section{Conclusions}

Cardiometabolic diseases have rapidly increased in the incidence and prevalence, in the last three decades to epidemic proportions worldwide. Obesity in children and adolescents has increased by ten-fold in the last four decades. During the same period, type-2 diabetes has doubled and obesity in adults has increased four-fold. No country has arrested, reversed, or prevented the increase in the incidence of these chronic diseases. Modern medicine has failed to develop a robust preventive medicine strategy. However, there is an increasing awareness of this situation and growing interest in developing novel strategies, to address this public health menace. Modern concepts of healthcare include an emphasis on overall wellness rather than just the absence of disease. Wellness score includes the valuation of physical activity, healthy diet, and purpose of life (62). Revolution in the emerging technologies has created an unlimited opportunity for entrepreneurs, to develop a variety of wearables, smart collating and computing platforms, health Apps and health portals.

Professional societies and global health platforms, celebrate World Diabetes Day and World Prematurity Day in November, to create awareness about type- 2 diabetes and prematurity. We have gone one step further, by describing the relationship between these two conditions. Furthermore, we have discussed some novel approaches, to address this global public health menace. Modern concepts in healthcare will pay greater importance, to the overall wellness of an individual. Overall wellness, depends upon physical fitness, healthy diets, and active life, with some achievable goals and purpose. Because of the fact, major chronic metabolic diseases are the manifestation of poor lifestyle, there is a great need to create awareness of these observations at the individual levels, as well as at the population level. As multinational experts have suggested, "governments in concert with the private sector, need to set policies, that promote healthy nutritional and agricultural policies, favor modifications in the environment that encourage physical activity, and make prevention affordable for all citizens at high risk."

\section{References}

1. Fuster V (2014) Global burden of cardiovascular disease (Editorial): J Amer Coll Cardiol 64: 520-522.

2. NCD Risk Factor Collaboration (NCD-RisC) (2017) Worldwide trends in blood pressure from 1975 to 2015: a pooled analysis of 1479 population-based measurement studies with 19.1 million participants. Lancet 389: P3755.

3. Global Burden of Metabolic Risk Factors for Chronic Disease Collaboration (2014) Cardiovascular disease, chronic kidney disease, and diabetes mortality burden of cardiometabolic risk factors from 1980 to 2010: a comparative risk assessment. Lancet Diab Endocrinol 2: 634-647.

4. NCD Risk Factor Collaboration (2005) Worldwide trends in diabetes since 1980: a pooled analysis of 751 population-based studies with 4.4 million participants. Lancet 387: 1513-1530.

5. Kearnery PM, Whelton M, Reynolds K (2005) Global burden of hypertension: analysis of worldwide data. Lancet 365: 217-223.

6. Evans A, Tolonen H, Hense HW (2001) Trends in coronary artery disease risk factors in the WHO MONICA project. Int. J. Epidemiol 30: S35-S40.

7. Juonala M, Vikari JS, Hurti-Kahonen B (2004) The 21year follow up of the cardiovascular risk in young Finns Study: risk factor levels, secular trends and east-west difference. J Intern Med 255: 457-468. 
8. Reilly JJ, El-Hamdouchi A, Diouf A (2018) Determining the worldwide prevalence of obesity. Lancet 391: P17731774.

9. NCD Risk Factor Collaboration (NCD-RisC) (2017) Worldwide trends in body mass-index, underweight, overweight, and obesity from 1975 to 2016: a pooled analysis of 2416 population-based measurement studies in 128.9 million children, adolescents, and adults. Lancet 390: $2627-2642$.

10. NCD Risk Factor Collaboration (NCD-RisC) (2016) Trends in adult body-mass index in 200 countries from 1975 to 2014: a pooled analysis of 1968 populationbased measurement studies with 19.2 million participants. Lancet 387: P1377-1396.

11. The Global Burden of Metabolic Risk Factors for Chronic Diseases Collaboration (BMI Mediate Effects) (2014) Metabolic mediators of the effects of body-mass index, overweight, and obesity on coronary artery disease and stroke: a pooled analysis of 97 prospective cohorts with 1.8 million participants. Lancet 383: 970-983.

12. WHO (2013) Global action plan for the prevention and control of non-communicable diseases 2013-2020? World Health Organization, Geneva, Switzerland.

13. Kontis V, Mathers CD, Rehm J (2014) Contribution of six risk factors to achieving the $25 \times 25$ non-communicable disease mortality reduction target: a modeling study. Lancet 384: 427-437.

14. Ng M, Fleming T, Robinson M (2014) Global, regional and national prevalence of overweight and obesity in children and adults during 1980-2013: a systematic analysis for the Global Burden of Disease Study 2013. Lancet 384: 766781.

15. NCD Risk Factor Collaboration (NCD-RisC) (2016) Worldwide trends in diabetes since 1980: a pooled analysis of 751 population-based studies with 4.4 million participants. Lancet 387: P1513-1530.

16. Seuring T, Archangelidi O, Suhrcke M (2015) The economic costs of type- 2 diabetes: a global systematic review. Pharmacoeconomics 33: 811-831.

17. Danaei G, Finucane MM, Lu Y (2011) National, regional, and global trends in fasting glucose and diabetes prevalence since 1980: systematic analysis of health examination surveys and epidemiological studies with 370 countryyears and 2.7 million participants. Lancet 378: 31-40.

18. Beagley J, Guariguata L, Weil C (2014) Global estimates of undiagnosed diabetes in adults. Diab Res. Clin Pract 103: $150-160$.

19. Gakidou E, Mallinger L, Abott-Klafter J (2011) Management of diabetes and associated cardiovascular risk factors in seven countries: a comparison of data from national health surveys. Bull World Health Organ 89: 172183.
20. Shen X, Vaidya A, Wu S (2016)The diabetes epidemic in China: An integrated review of national surveys. Endocr Pract 22: 1119-1129.

21. World Health Organization (2016) Global burden of diseases (NCDs). Report prepared by the institute of health metrics and evaluation (IHME). Global Burden of Disease Report, Global Burden of Disease, The Lancet, USA.

22. Sasson C, Eckel R, Alger H (2018) American Heart Association Diabetes and Cardiometabolic Health Summit and Recommendations. Am Heart Assoc 7: e009271.

23. Negrato CA, Gomes MB (2013) Low birth weight: Causes and consequences. Diab, and Metabolism 5: 49.

24. Barker DJP (1997) Maternal Nutrition, Fetal Nutrition and Disease in Later Life. Nutrition 13: 807.

25. Hales CN Barker DJP (1992) Type-2 (non-insulin dependent) diabetes mellitus: the thrifty phenotype hypothesis. Diabetologia 35:595-601.

26. Wells JCK (2007) The thrifty phenotype as an adaptive maternal effect. Biol Rev 82: 143-172.

27. Robinson R (2001) The fetal origins of adult disease. No longer just a hypothesis and may be critically important in South Asia. BMJ 322: 375-376.

28. Barker DJP (1992) Fetal origins of adult disease. BMJ Books London. ISBN \#0-7279-0743-3, 1992.

29. Veena SR, Geetha S, Leary SD (2007) Relationship of maternal and paternal birth weights to features of the metabolic syndrome in the adult offspring: An integrational Study in South India. Diabetologia 50: 43-54.

30. Krishnaveni GV, Hill JC, Leary SD (2005) Anthropometry, glucose intolerance and insulin concentrations in Indian Children: relationship s to maternal glucose and insulin concentrations during pregnancy. Diabetes Care 28:29192925.

31. Kanade AN, Rao S, Kelkar RS (2008) Maternal nutrition and birth size among urban affluent and rural women in India. J Amer Coll Nutr 27: 137-145.

32. Low birth weight (2005) Country, regional and global estimates. WHO 2005. Reproductive Health Documentation 54 .

33. Barker DJP (1994) Outcome of Low Birth Weight. Horm Res 42: 223-230.

34. Editorials: The relation between fetal malnutrition and chronic disease later in life: Good nutrition and lifestyle matter from womb to tomb. BMJ 315: 825-826.

35. Ramakrishnan U (2004) Nutrition and low birth weight: from research to practice. Am. J. Clin. Nutr. 79: 17-21.

36. WHO Maternal anthropometry and pregnancy outcomes (1995) a WHO Collaborative Study. Bull. WHO 73: 1-98. 
37. George Dover (2009) The Barker Hypothesis: How Pediatricians will diagnose and Prevent Common AdultOnset Diseases. Trans Am Clin Climatol. Assoc 120: 199207.

38. Freishtat R (2018) Maternal Adipocyte-derived Exosomes in the Thin-Fat Baby Paradox. Fogarty International Research Grant, National Institutes of Health (NIH), 1R21 HD094127-01. 2018-2020.

39. Yajnik CS, Fall CH, Coyaji KJ (2003) Neonatal anthropometry: the thin-fat Indian baby. The Pune Maternal Nutrition Study. Int J Obes Relat Disord 27: $173-80$

40. Brandon AE, Liao BM, Diakanastasis B (2019) Protein Kinase $\mathrm{C}$ epsilon in adipose tissue, but not liver improves glucose tolerance. Cell Metab 29: 183-191.e7.

41. Bjerregaard LG, Jensen BW, Angquist L (2018) Change in overweight from childhood to early adulthood and risk for type- diabetes. N Engl J Med 378: 1302-1312.

42. Tabak AG, Herder C, Ratmann W (2012) Prediabetes: a high-risk state for diabetes development. Lancet Diab Ser 379: 2279-2290.

43. Yip WCY, Sequeira IR, Plank LD (2017) Prevalence of Prediabetes across ethnicities: A review of Impaired Fasting Glucose (IFG) and Impaired Glucose tolerance (IGT) for classification of Dysglycemia. Nutrients 9: 1273.

44. Bergman, Buysschaert M, Schwartz PEH (2012) Diabetes prevention: global health policy and perspectives from the ground. Diab Manag. (Lond) 2: 309-321.

45. Rao GHR (2018) Diagnosis of early risks, management of risks, and reduction of vascular diseases. J. Clin Cardiol and Diagn 1: 1-10.

46. Rao GHR (2018) Prevention or reversal of cardiometabolic diseases. J Clin Prevent Cardiol 7: 22-28.

47. Rao GHR (2015) Non-traditional approaches to diagnosis and management of type- 2 diabetes mellitus. Point of View. J Diab Metabol 6: 489.

48. Herman WH (2017) The global agenda for the prevention of type-2 diabetes. Nutr Re 75: 13-18.

49. Puska P (2002) Successful prevention of noncommunicable diseases: 25-year experience with North Karelia project in Finland Publ. Health Med 4: 5-7.

50. Bai Y, Hibbing P, Mantis C (2017) Comparative evaluation of heart rate-based monitors: Apple Watch vs Fitbit Charge HR. J of Sports Sci 36: 1-8.

51. Henriksen A, Mikalsen MH, Woldaregay AZ (2018) Using fitness trackers and smartwatches to measure physical activity in research: Analysis of consumer wrist-worn wearables. J Med Internet Res 20: e110.

52. Emaus A, Degerstrom J, Wilsgaard T (2010) Does a variation in objectively measured physical activity, resting heart rate, and physical fitness? Results from the Tromso Study. Scand. J Publ Health 38: 105-118.

53. The All of Us Research Program Investigators (2019) The "All of Us" Research program. New Engl J Med 391: 668676.

54. Gandhi PG, Rao GHR (2014) The Spectral Analysis of Photoplethysmography to Evaluate an Independent Cardiovascular Risk Factor. Int J Gen Med 7: 39-47.

55. Gandhi PG, Rao GHR (2015) Detection of Neuropathy Using SudoMotor Tests in Type-2 Diabetes. Degenerative Neurological and Neuromuscular Diseases 5: 1-7.

56. Maarek A, Gandhi PG, Rao GHR (2015) Identifying autonomic neuropathy and endothelial dysfunction in Type 11 diabetic patients. EC Neurology 22: 63-78.

57. Rao GHR, Gandhi PG (2014) Need for a non-invasive diagnostic platform for early detection and management of cardiometabolic disorders. J. Clin Prevent. Cardiol 3: 93-98.

58. Rajesh B, Hussain R, Giridhar A (2016) Autofluorescence and Infrared Fundus Imaging for Detection of Retinal Emboli and Unmasking Undiagnosed Systemic Abnormalities. J Opththalm Vis Res 11: 449-451.

59. Jasti N, Bhargav H, Sinha S (2019) Medical applications of Infrared thermography: A narrative review. J of Stem Cells 14: 35-53.

60. Yusuf S, Hawken S, Ounpuu S (2004) Effect of potentially modifiable risk factors associated with myocardial infraction in 52 countries (the INTERHEART study): Case-control study. Lancet 364: 937-952.

61. Khera AV, Emdin CA, Drake I (2016) Genetic risk, adherence to a healthy lifestyle, and coronary artery disease. N Engl J Med 375: 2349-2358.

62. Malmefeldt E Rao (2019.), GHR: Changin Concepts of Healthcare: Physical Activity, Fitness, and Wellness. EC Endocrinology and Metabolic Research. 4 (6): 238-250.

Copyright: (C)2020 Gundu HR Rao. This is an open-access article distributed under the terms of the Creative Commons Attribution License, which permits unrestricted use, distribution, and reproduction in any medium, provided the original author and source are credited. 